\title{
Transient deformation of a poroelastic channel bed
}

\author{
P. C. Hsieh ${ }^{\mathrm{a},}$, W. P. Shih ${ }^{\mathrm{b}}$ and L. H. Huang ${ }^{\mathrm{b}}$ \\ ${ }^{a}$ Department of Soil and Water Conservation, National Chung-Hsing University, Taichung 40227, Taiwan, ROC \\ ${ }^{\mathrm{b}}$ Department of Civil Engineering, National Taiwan University, Taipei 106, Taiwan, ROC
}

\begin{abstract}
SUMMARY
The coupled transient response of a poroelastic bed form due to stream flow and non-linear water waves is investigated numerically. The theory of potential flow is applied to channel flow while Biot's theory of poroelasticity (J. Appl. Phys. 1962; 33(4):1482) is adopted to deal with the deformable porous bed. A boundary-fitted co-ordinate system is used to calculate the variation in the bed form. The result of a simple periodic wave form over a soft poroelastic bed agrees well with the analytical solution of Hsieh et al. (J. Eng. Mech., ASCE 2000; 126(10):1064). However, due to the rapidly damping second dilatational wave inside the soft poroelastic bed, the solution for transient bed form near the interface is not easy to compute accurately. In order to overcome this difficulty, a simplified numerical model based on the boundary layer correction concept proposed by Hsieh et al. (2000) is established, which neglects Darcy's terms. The transient deformation of an irregular poroelastic bed that includes a trench and a downward step at the channel bed is simulated successfully. Copyright (C) 2002 John Wiley \& Sons, Ltd.
\end{abstract}

KEY WORDS: Transient deformation; soft poroelastic bed; boundary layer correction concept; boundaryfitted co-ordinate transformation

\section{INTRODUCTION}

The problem of flow past a porous deformable bed is always an important topic to hydraulic and ocean engineers. In addition to the complexity of interrelated mechanics between homogeneous water flow and porous deformable bed, the interaction between the skeleton and the pore-saturated fluid of the porous bed is even more complicated. For these subjects, some researches based on experiments [1-3] or simplified theories, which may apply empirical mass transport formulas [4-7] to avoid the analysis of complicated deformable porous media mechanics, were published to explain the physical behaviour. In addition, Darwin [8] conducted experiments on sand-ripple caused by oscillatory bed motion and concluded that the eddies induced by a series of vortex acting on the sandy bed would probably render unstable ripple into stable state. Bagnold [9] explained that the instability of dune in the desert was due to the impact

\footnotetext{
${ }^{\dagger}$ Correspondence to: P. C. Hsieh, Department of Soil and Water Conservation, National Chung-Hsing University, Taichung 40227, Taiwan, R.O.C.

E-mail: pchsieh@dragon.nchu.edu.tw

Contract/grant sponsor: National Science Council of the Republic of China; contract/grant numbers: NSC 86-2621E002-016 and NSC 88-2611-E-002-028.
}

Copyright (C) 2002 John Wiley \& Sons, Ltd.

Received 12 June 2001 Revised 30 May 2002 
of sand by wind. Exner [10] established a differential erosion equation for two-dimensional flow, which indicated that the change in bed elevation was due to dilatational variation of bottom velocity. Anderson [11] applied Exner's [10] erosion equation to explain the mechanism of ripple formation. However, the mechanism of dune and antidune formation is somewhat different from that of ripple. Kennedy [12] applied an empirical sediment transport formula to govern the continuity of the porous bed. Also, he used instability analysis of potential theory to obtain his famous discovery about the dune and antidune formations in alluvial channels. Unfortunately, owing to the constraint of instability analysis, Kennedy [12] could only find the dominant wavelength of stable bed forms instead of the whole bed form.

In fact, fluid within porous material interacting with a deforming solid skeleton is a more complicated two-phase flow problem for a realistic analysis. Biot [13] developed a poroelasticity theory to describe elastic waves in a fluid saturated porous solid. Mei and Foda [14] proposed a boundary layer correction to simplify the analysis; however, their approach was based on physical intuition but without systematic analysis. By treating the bed as a poroelastic material, Huang and Song [15] solved the problem of oscillatory linear water waves interacting with a deformable bed by using three decoupled Helmoltz equations derived by Huang and Chwang [16]. In their solution, five non-dimensional parameters were derived. Chen et al. [17] also applied Huang and Chwang's [16] approach along with the conventional Stokes expansion of deep water waves based on the perturbation parameter $\varepsilon_{1}=k_{0} a\left(k_{0}\right.$ and $a$ are the wave number and the amplitude of incoming water wave, respectively) to investigate the dynamic response of permeable bed material to non-linear water waves. They found that the conventional Stokes expansion is only valid for hard poroelastic bed material but invalid for soft materials even though the Ursell parameter is small. (Here 'hard' and 'soft' are defined according to a parameter $\Pi$, a Mach number of the second longitudinal wave inside the poroelastic material, in their paper.) Huang and Chiang [18] simulated stable bed forms under a constant current and linear oscillatory water waves by an approach similar to that of Huang and Song [15]. In Huang and Chiang [18], various bed forms such as dune, antidune and flat bed under linear oscillatory water wave accompanied with a constant current were obtained. The ambiguous lagged distance $\delta$, introduced in Kenney [12], which has bothered researchers in river mechanics for many years was clarified. Huang et al. [19] and Hsieh et al. [20] used a two-parameter $\left(\varepsilon_{1}, \varepsilon_{2}=k_{0} / k_{2}, k_{2}\right.$ is the wave number of the second longitudinal wave inside the poroelastic material) perturbation expansion to discuss the dynamic response of soft poroelastic bed to linear and non-linear oscillatory water waves by a boundary layer correction approach.

The aforementioned studies are mostly focused on the stable bed form problem, however, the transient response of a constant current flowing over a semi-infinite soft poroelastic deforming bed along with non-linear water waves (Figure 1) remains to be analysed. In the present study, a finite difference numerical model based on the poroelastic theory is proposed to discuss the transient bed form.

\section{FORMULATION}

This study analyses the problem of a non-linear water wave along with a uniform channel flow over a poroelastic deformable bed, as defined schematically in Figure 1. Region 1 is homogeneous water, considered as potential flow, while region 2 is a semi-infinite porous medium saturated with water governed by Biot's theory of poroelasticity [21]. The co-ordinates 


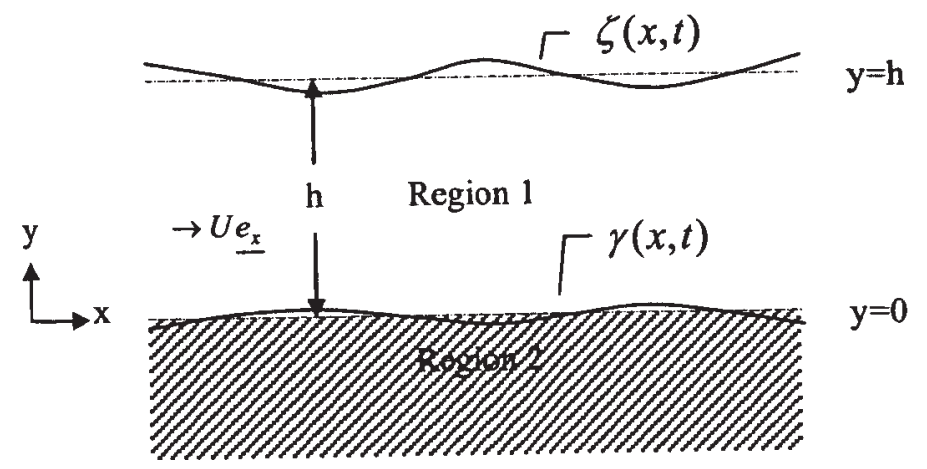

Figure 1. Definition sketch.

of region 1 range from $y=\gamma(x, t)$ to $y=h+\zeta(x, t)$, and region 2 from $y=\gamma(x, t)$ to $y \rightarrow-\infty$. The symbols $\zeta$ and $\gamma$ represent the displacements of waves from the mean free surface $(y=h)$ and mean bed interface $(y=0)$, respectively.

\subsection{Boundary value problem}

Assuming that the homogeneous channel flow is a potential flow, the flow velocity can be written as a given constant current $(U)$ in the $x$ direction (i.e. $U \underline{e}_{x}$ ) plus a perturbed velocity $\underline{u}_{1}$. Thus the perturbed velocity potential $\phi_{1}$ satisfies

$$
\nabla \phi_{1}=\underline{u}_{1}
$$

The equations of continuity and momentum in terms of velocity potential $\phi_{1}$ become

$$
\begin{gathered}
\nabla^{2} \phi_{1}=0 \\
\frac{\partial \phi_{1}}{\partial t}+\frac{1}{2}\left[2 U \frac{\partial \phi_{1}}{\partial x}+\left(\frac{\partial \phi_{1}}{\partial x}\right)^{2}+\left(\frac{\partial \phi_{1}}{\partial y}\right)^{2}\right]+\frac{P_{1}}{\rho_{\mathrm{f}}}=0
\end{gathered}
$$

where $P_{1}$ is the perturbed pressure, and $\rho_{\mathrm{f}}$ is the water density.

Referring to Biot's theory of poroelasticity [13], the linear momentum equations of solid skeleton and fluid for the porous bed may be written as

$$
\begin{aligned}
& \nabla \cdot \underline{\underline{\sigma}}=\rho_{11} \frac{\partial^{2} \underline{d}}{\partial t^{2}}+\rho_{12} \frac{\partial^{2} \underline{D}}{\partial t^{2}}+b\left(\frac{\partial \underline{d}}{\partial t}-\frac{\partial \underline{D}}{\partial t}\right) \\
& \nabla \cdot \underset{\underline{S}}{ }=\rho_{12} \frac{\partial^{2} \underline{d}}{\partial t^{2}}+\rho_{22} \frac{\partial^{2} \underline{D}}{\partial t^{2}}-b\left(\frac{\partial \underline{d}}{\partial t}-\frac{\partial \underline{D}}{\partial t}\right)
\end{aligned}
$$

with

$$
\begin{aligned}
& \underline{\underline{\sigma}}=\underline{\underline{\tau}}-\left(1-n_{0}\right) P_{2} \underline{\underline{I}} \\
& \underline{\underline{\tau}}=2 G \underline{\underline{e}}+\lambda(\nabla \cdot \underline{d}) \underline{\underline{I}}
\end{aligned}
$$




$$
\begin{gathered}
\underline{\underline{e}}=\frac{1}{2}\left[\nabla \underline{d}+(\nabla \underline{d})^{t}\right] \\
\underline{\underline{S}}=-n_{0} P_{2} \underline{\underline{I}} \\
\rho_{11}=\left(1-n_{0}\right) \rho_{\mathrm{s}}+\rho_{\mathrm{a}} \\
\rho_{12}=-\rho_{\mathrm{a}} \\
\rho_{22}=n_{0} \rho_{\mathrm{f}}+\rho_{\mathrm{a}} \\
b=\mu n_{0}^{2} / k_{\mathrm{p}}
\end{gathered}
$$

where $\underline{\underline{\sigma}}$ is the solid stress tensor, $\tau$ the effective stress tensor of the solid, $\underline{\underline{e}}$ the strain tensor of solid, $\underset{\underline{S}}{\underline{\underline{S}}}$ the normal stress tensor of the fluid, $\underline{d}$ and $\underline{D}$ are the solid and fluid displacement vectors, respectively, $P_{2}$ the perturbed pressure of the fluid inside the porous medium; $\rho_{\mathrm{s}}$ the solid density; $\rho_{\mathrm{a}}$ the mass coupling effect (neglected in this study), $n_{0}$ the porosity, $\mu$ the fluid viscosity, $k_{\mathrm{p}}$ the specific permeability, $G$ and $\lambda$ are the Lame's constants of elasticity and $I \underline{\underline{I}}$ the identity matrix.

Combining the continuity equations of the solid and the fluid with the state equation of the fluid, and after linearization of the porosity (see Reference [22]), we find

$$
\frac{\partial P_{2}}{\partial t}=-\frac{K}{n_{0}}\left[\left(1-n_{0}\right) \nabla \cdot\left(\frac{\partial \underline{d}}{\partial t}\right)+n_{0} \nabla \cdot\left(\frac{\partial \underline{D}}{\partial t}\right)\right]
$$

for the perturbed pressure. In Equation (14), $K$ is the bulk modulus of compressibility of fluid inside the porous bed.

According to Huang and Chwang [16], the displacement vectors of skeleton and fluid could be separated into dilatational and rotational parts

$$
\begin{aligned}
& \underline{d}=\nabla \varphi_{d}+\nabla \times \underline{H}_{d} \\
& \underline{D}=\nabla \varphi_{D}+\nabla \times \underline{H}_{D}
\end{aligned}
$$

The first terms on the right-hand sides of Equations (15) and (16) represent the dilatational components (two dilatational waves) of the skeleton and fluid displacements, respectively; while the second terms represent rotational components of the skeleton and fluid displacements. The former ones are irrotational while the latter ones are solenoidal. Referring to Morse and Feshbach [23], $\underline{H}_{d}$ and $\underline{H}_{D}$ can be expressed as

$$
\underline{H}_{d}=\omega_{d} \underline{\underline{e}} \underline{\text { and }} \quad \underline{H}_{D}=\omega_{D} \underline{e}_{Z}
$$

where $\underline{e}_{z}$ is the unit vector normal to the $x-y$ plane.

Substituting Equations (15)-(17) into Equations (4) and (5), we obtain

$$
\begin{gathered}
(2 G+\lambda) \nabla^{2} \varphi_{d}-\left(1-n_{0}\right) P_{2}=\rho_{11} \frac{\partial^{2} \varphi_{d}}{\partial t^{2}}+\rho_{12} \frac{\partial^{2} \varphi_{D}}{\partial t^{2}}+b\left(\frac{\partial \varphi_{d}}{\partial t}-\frac{\partial \varphi_{D}}{\partial t}\right) \\
-n_{0} P_{2}=\rho_{12} \frac{\partial^{2} \varphi_{d}}{\partial t^{2}}+\rho_{22} \frac{\partial^{2} \varphi_{D}}{\partial t^{2}}-b\left(\frac{\partial \varphi_{d}}{\partial t}-\frac{\partial \varphi_{D}}{\partial t}\right)
\end{gathered}
$$




$$
\begin{gathered}
G \nabla^{2} \omega_{d}=\rho_{11} \frac{\partial^{2} \omega_{d}}{\partial t^{2}}+\rho_{12} \frac{\partial^{2} \omega_{D}}{\partial t^{2}}+b\left(\frac{\partial \omega_{d}}{\partial t}-\frac{\partial \omega_{D}}{\partial t}\right) \\
0=\rho_{12} \frac{\partial^{2} \omega_{d}}{\partial t^{2}}+\rho_{22} \frac{\partial^{2} \omega_{D}}{\partial t^{2}}-b\left(\frac{\partial \omega_{d}}{\partial t}-\frac{\partial \omega_{D}}{\partial t}\right)
\end{gathered}
$$

Substituting Equations (15)-(17) into Equation (14), we get

$$
\frac{\partial P_{2}}{\partial t}=-\frac{K}{n_{0}} \frac{\partial}{\partial t}\left[\left(1-n_{0}\right) \nabla^{2} \varphi_{d}+n_{0} \nabla^{2} \varphi_{D}\right]
$$

Equations (18)-(22) are the governing equations.

There are three boundaries that require boundary conditions. They are (i) the free surface $[y=h+\zeta(x, t)]$, (ii) the channel bed $[y=\gamma(x, t)]$, and (iii) the deep far field of the porous bed $[y \rightarrow-\infty]$.

On the free surface, the kinematic boundary condition is

$$
\frac{\partial \phi_{1}}{\partial y}=\frac{\partial \zeta}{\partial t}+\frac{\partial \zeta}{\partial x}\left(U+\frac{\partial \phi_{1}}{\partial x}\right)
$$

and the dynamic boundary condition is

$$
\frac{\partial \phi_{1}}{\partial t}+\frac{1}{2}\left[2 U \frac{\partial \phi_{1}}{\partial x}+\left(\frac{\partial \phi_{1}}{\partial x}\right)^{2}+\left(\frac{\partial \phi_{1}}{\partial y}\right)^{2}\right]+g \zeta=0
$$

On the porous bed, the continuity of pressure implies that

$$
P_{1}=P_{2}
$$

while the continuity of fluid flux gives

$$
\begin{aligned}
\frac{\partial \phi_{1}}{\partial y}-\frac{\partial \gamma}{\partial x} \frac{\partial \phi_{1}}{\partial x}= & U \frac{\partial \gamma}{\partial x}+\frac{\partial}{\partial t}\left[\left(1-n_{0}\right)\left(\frac{\partial \varphi_{d}}{\partial y}-\frac{\partial \omega_{d}}{\partial x}\right)+n_{0}\left(\frac{\partial \varphi_{D}}{\partial y}-\frac{\partial \omega_{D}}{\partial x}\right)\right. \\
& \left.-\left(1-n_{0}\right) \frac{\partial \gamma}{\partial x}\left(\frac{\partial \varphi_{d}}{\partial y}-\frac{\partial \omega_{d}}{\partial x}\right)-n_{0} \frac{\partial \gamma}{\partial x}\left(\frac{\partial \varphi_{D}}{\partial y}-\frac{\partial \omega_{D}}{\partial x}\right)\right] .
\end{aligned}
$$

The continuity of effective stress of the solid, i.e. $\underline{n_{2}} \cdot \underline{\underline{\tau}}=\underline{0}$, gives

$$
\begin{gathered}
G\left(2 \varphi_{d, x y}+\omega_{d, y y}-\omega_{d, x x}\right)-\frac{\partial \gamma}{\partial x}\left[(2 G+\lambda) \varphi_{d, x x}+2 G \omega_{d, y x}+\lambda \varphi_{d, y y}\right]=0 \\
(2 G+\lambda) \varphi_{d, y y}+2 G \omega_{d, x y}+\lambda \varphi_{d, x x}-\frac{\partial \gamma}{\partial x} G\left(2 \varphi_{d, x y}+\omega_{d, y y}-\omega_{d, x x}\right)=0
\end{gathered}
$$

At the far field of the porous bed, i.e. $y \rightarrow-\infty$, the boundary conditions consist of vanishing displacement vectors, i.e.

$$
\lim _{y \rightarrow-\infty}\left\{\phi_{1}, \varphi_{d}, \varphi_{D}, \omega_{d}\right\}=0
$$


and no perturbation is propagated to the upstream and downstream boundaries, i.e.

$$
\begin{array}{ll}
\lim _{x \rightarrow \pm \infty} \frac{\partial}{\partial n}\left\{\varphi_{d}, \varphi_{D}, \omega_{d}\right\}=0 & \\
\lim _{x \rightarrow \pm \infty} \begin{cases}\frac{\partial \phi_{1}}{\partial n} \pm \mathrm{i} k \phi_{1}=0, & \text { periodic wave problem } \\
\frac{\partial \phi_{1}}{\partial n}=0, & \text { transient wave problem }\end{cases}
\end{array}
$$

In addition, the kinematic boundary condition at the porous bed interface results in

$$
\frac{\partial \gamma}{\partial t}=\frac{\partial}{\partial t}\left[\frac{\partial \varphi_{d}}{\partial y}-\frac{\partial \omega_{d}}{\partial x}-\frac{\partial \gamma}{\partial x}\left(\frac{\partial \varphi_{d}}{\partial x}+\frac{\partial \omega_{d}}{\partial y}\right)\right]
$$

which allows to determine the surface elevation of the porous bed, $\gamma$.

The governing equations (2), (18)-(21) with perturbed pressure in porous bed, Equation (22), surface elevations $\zeta$ and $\gamma$, Equations (24) and (31), together with other boundary conditions, Equations (23), (25)-(30), and a properly 'warm-up' initial condition form a well-posed problem.

\subsection{Non-dimensionalization through boundary layer correction}

When the poroelastic bed is composed of hard material, the inertial terms of the governing equations (first two terms on the right-hand side of Equations (18)-(21)) are unimportant and they can be neglected (see Reference [24]). The deformation is also negligible. However, when the poroelastic material is soft, the inertial terms are important, and the second dilatational wave travelling through the porous bed has a much shorter wavelength than that of the wave travelling through homogeneous water (see Reference [17]). Thus there exists a small boundary layer near the interface, inside the soft poroelastic bed. Because the effect of the boundary layer will render errors of the partial derivative in the vertical direction for the second longitudinal wave, the traditional estimation of the deformable bed by Stokes expansion based on $\varepsilon_{1}$ will fail [17]. Hsieh et al. [20] proposes a boundary layer correction approach based on multiple length scales to overcome this difficulty. Since we are interested in bed form deformation in this study, the following discussion will concentrate on soft poroelastic material only.

The coefficients of the governing equations, Equations (18)-(21), range too far to be solved directly by numerical computation so that non-dimensionalization is needed to reduce the difference of the magnitude of each term. We define

$$
\begin{gathered}
x=L x^{*}, \quad y=L y^{*} \\
\phi_{1}=L \sqrt{g L} \phi_{1}^{*}, \quad t_{1}=\sqrt{L / g} t_{1}^{*} \\
\varphi_{d}=L^{2} \varphi_{d}^{*}, \quad \omega_{d}=L^{2} \omega_{d}^{*} \\
\varphi_{D}=L^{2} \varphi_{D}^{*}, \quad \omega_{D}=L^{2} \omega_{D}^{*} \\
P_{2}=-\frac{K}{n_{0}} P_{2}^{*}, \quad t_{2}=\sqrt{L / g} t_{2}^{*}
\end{gathered}
$$


In which, all symbols with an asterisk stand for the dimensionless variables. The order of $t_{2}^{*}$ is found to be $O(1)$. Equations (2) and (18)-(22) can be rewritten as

$$
\begin{gathered}
\hat{\alpha}_{1} \phi_{1, \xi \xi}^{* n}+2 \hat{\alpha}_{2} \phi_{1, \xi \eta}^{*{ }_{n}}+\hat{\alpha}_{3} \phi_{1, \eta \eta}^{*}+\hat{\alpha}_{4} \phi_{1, \xi}^{* n}+\hat{\alpha}_{5} \phi_{1, \eta}^{*}=0 \\
\frac{(2 G+\lambda)}{K} \nabla_{*}^{2} \varphi_{d}^{*}-\left(1-n_{0}\right) P_{2}^{*}=\frac{\rho_{11} g L}{K} \frac{\partial^{2} \varphi_{d}^{*}}{\partial t^{* 2}}+\frac{\rho_{12} g L}{K} \frac{\partial^{2} \varphi_{D}^{*}}{\partial t^{* 2}} \\
+b \sqrt{\frac{g}{L}} \frac{L^{2}}{K}\left(\frac{\partial \varphi_{d}^{*}}{\partial t^{*}}-\frac{\partial \varphi_{D}^{*}}{\partial t^{*}}\right) \\
-n_{0} P_{2}^{*}=\frac{\rho_{12} g L}{K} \frac{\partial^{2} \varphi_{d}^{*}}{\partial t^{* 2}}+\frac{\rho_{22} g L}{K} \frac{\partial^{2} \varphi_{D}^{*}}{\partial t^{* 2}}-b \sqrt{\frac{g}{L}} \frac{L^{2}}{K}\left(\frac{\partial \varphi_{d}^{*}}{\partial t^{*}}-\frac{\partial \varphi_{D}^{*}}{\partial t^{*}}\right) \\
\frac{G}{K} \nabla_{*}^{2} \omega_{d}^{*}=\frac{\rho_{11} g L}{K} \frac{\partial^{2} \omega_{d}^{*}}{\partial t^{* 2}}+\frac{\rho_{12} g L}{K} \frac{\partial^{2} \omega_{D}^{*}}{\partial t^{* 2}}+b \sqrt{\frac{g}{L}} \frac{L^{2}}{K}\left(\frac{\partial \omega_{d}^{*}}{\partial t^{*}}-\frac{\partial \omega_{D}^{*}}{\partial t^{*}}\right) \\
0=\frac{\rho_{12} g L}{K} \frac{\partial^{2} \omega_{d}^{*}}{\partial t^{* 2}}+\frac{\rho_{22} g L}{K} \frac{\partial^{2} \omega_{D}^{*}}{\partial t^{* 2}}-b \sqrt{\frac{g}{L}} \frac{L^{2}}{K}\left(\frac{\partial \omega_{d}^{*}}{\partial t^{*}}-\frac{\partial \omega_{D}^{*}}{\partial t^{*}}\right) \\
\frac{\partial P_{2}^{*}}{\partial t^{*}}=-\frac{1}{n_{0}} \frac{\partial}{\partial t^{*}}\left[\left(1-n_{0}\right) \nabla_{*}^{2} \varphi_{d}^{*}+n_{0} \nabla_{*}^{2} \varphi_{D}^{*}\right]
\end{gathered}
$$

in which, the coefficients $\hat{\alpha}_{1}, \hat{\alpha}_{2}, \hat{\alpha}_{3}, \hat{\alpha}_{4}$ and $\hat{\alpha}_{5}$ are referred in Appendix A.

Adding Equation (38) to Equation (39) to eliminate Darcy's terms, we get

$$
\frac{(2 G+\lambda)}{K} \nabla_{*}^{2} \varphi_{d}^{*}-P_{2}^{*}=\frac{\left(\rho_{11}+\rho_{12}\right) g L}{K} \frac{\partial^{2} \varphi_{d}^{*}}{\partial t^{* 2}}+\frac{\left(\rho_{12}+\rho_{22}\right) g L}{K} \frac{\partial^{2} \varphi_{D}^{*}}{\partial t^{* 2}}
$$

and similarly adding Equation (40) to Equation (41), we have

$$
-\frac{G}{K} \nabla_{*}^{2} \omega_{d}^{*}=\frac{\left(\rho_{11}+\rho_{12}\right) g L}{K} \frac{\partial^{2} \omega_{d}^{*}}{\partial t^{* 2}}+\frac{\left(\rho_{12}+\rho_{22}\right) g L}{K} \frac{\partial^{2} \omega_{D}^{*}}{\partial t^{* 2}}
$$

The huge coefficient $b$ of Darcy's terms in Equation (39) is not suitable for numerical computation. However, for soft poroelastic material the magnitude of the second longitudinal wave is rather small and the displacement potentials of solid and fluid are very close, i.e. $\varphi_{d}^{*} \cong \varphi_{D}^{*}$ and $\omega_{d}^{*} \cong \omega_{D}^{*}$, by the boundary layer correction proposed by Hsieh et al. [20]. Since Darcy's terms are mainly due to the second longitudinal wave, we find that when the porous material is soft, Darcy's terms are negligible. We therefore can simplify Equations (39) and (41), respectively, to

$$
\begin{gathered}
-n_{0} \frac{K}{b L^{2}} \sqrt{\frac{L}{g}} P_{2}^{*}=\frac{\rho_{12}}{b} \sqrt{\frac{g}{L}} \frac{\partial^{2} \varphi_{d}^{*}}{\partial t^{* 2}}+\frac{\rho_{22}}{b} \sqrt{\frac{g}{L}} \frac{\partial^{2} \varphi_{D}^{*}}{\partial t^{* 2}} \\
0=\frac{\rho_{12}}{b} \sqrt{\frac{g}{L}} \frac{\partial^{2} \omega_{d}^{*}}{\partial t^{* 2}}+\frac{\rho_{22}}{b} \sqrt{\frac{g}{L}} \frac{\partial^{2} \omega_{D}^{*}}{\partial t^{* 2}}
\end{gathered}
$$

Note that Equations (39), (41), (43), (44) and (42) are the full non-dimensional governing equations for the soft porous bed, while Equations (43)-(46) and (42) are the simplified nondimensional governing equations for the soft porous bed. The non-dimensional boundary 
conditions are as follows:

1. Kinematic free surface boundary condition

$$
\begin{aligned}
\frac{\partial \phi_{1}^{*}}{\partial y^{*}}= & -\frac{1}{g}\left[\frac{\partial^{2} \phi_{1}^{*}}{\partial t^{* 2}}+2\left(U^{*}+\frac{\partial \phi_{1}^{*}}{\partial x^{*}}\right) \frac{\partial^{2} \phi_{1}^{*}}{\partial x^{*} \partial t^{*}}+\left(U^{*}+\frac{\partial \phi_{1}^{*}}{\partial x^{*}}\right) \frac{\partial^{2} \phi_{1}^{*}}{\partial x^{* 2}}\right. \\
& \left.+\frac{\partial \phi_{1}^{*}}{\partial y^{*}} \frac{\partial^{2} \phi_{1}^{*}}{\partial y^{*} \partial t^{*}}+\frac{\partial \phi_{1}^{*}}{\partial y^{*}}\left(U^{*}+\frac{\partial \phi_{1}^{*}}{\partial x^{*}}\right) \frac{\partial^{2} \phi_{1}^{*}}{\partial x^{*} \partial y^{*}}\right] .
\end{aligned}
$$

2. Dynamic free surface boundary condition

$$
\frac{\partial \phi_{1}^{*}}{\partial t^{*}}+\frac{1}{2}\left[2 U^{*} \frac{\partial \phi_{1}^{*}}{\partial x^{*}}+\left(\frac{\partial \phi_{1}^{*}}{\partial x^{*}}\right)^{2}+\left(\frac{\partial \phi_{1}^{*}}{\partial y^{*}}\right)^{2}\right]+\zeta^{*}=0
$$

3. Continuity of flux at water/porous bed interface

$$
\begin{aligned}
\frac{\partial \phi_{1}^{*}}{\partial y^{*}}-\frac{\partial \gamma^{*}}{\partial x^{*}} \frac{\partial \phi_{1}^{*}}{\partial x^{*}}= & U^{*} \frac{\partial \gamma^{*}}{\partial x^{*}}+\frac{\partial}{\partial t^{*}}\left[\left(1-n_{0}\right)\left(\frac{\partial \varphi_{d}^{*}}{\partial y^{*}}-\frac{\partial \omega_{d}^{*}}{\partial x^{*}}\right)\right. \\
& +n_{0}\left(\frac{\partial \varphi_{D}^{*}}{\partial y^{*}}-\frac{\partial \omega_{D}^{*}}{\partial x^{*}}\right)-\left(1-n_{0}\right) \frac{\partial \gamma^{*}}{\partial x^{*}}\left(\frac{\partial \varphi_{d}^{*}}{\partial y^{*}}-\frac{\partial \omega_{d}^{*}}{\partial x^{*}}\right) \\
& \left.-n_{0} \frac{\partial \gamma^{*}}{\partial x^{*}}\left(\frac{\partial \varphi_{D}^{*}}{\partial y^{*}}-\frac{\partial \omega_{D}^{*}}{\partial x^{*}}\right)\right] .
\end{aligned}
$$

4. Continuity of effective stresses (i.e. $\tau_{x y}=0$ and $\tau_{y y}=0$ ) at water/porous bed interface

$$
\begin{gathered}
\frac{G}{K}\left(2 \varphi_{d, x^{*} y^{*}}^{*}+\omega_{d, y^{*} y^{*}}^{*}-\omega_{d, x^{*} x^{*}}^{*}\right) \\
-\frac{\partial \gamma^{*}}{\partial x^{*}}\left[\frac{(2 G+\lambda)}{K} \varphi_{d, x^{*} x^{*}}^{*}+\frac{2 G}{K} \omega_{d, y^{*} x^{*}}^{*}+\frac{\lambda}{K} \varphi_{d, y^{*} y^{*}}^{*}\right]=0 \\
\frac{(2 G+\lambda)}{K} \varphi_{d, y^{*} y^{*}}^{*}-\frac{2 G}{K} \omega_{d, x^{*} y^{*}}^{*}+\frac{\lambda}{K} \varphi_{d, x^{*} x^{*}}^{*} \\
-\frac{\partial \gamma^{*}}{\partial x^{*}} \frac{G}{K}\left(2 \varphi_{d, x^{*} y^{*}}^{*}+\omega_{d, y^{*} y^{*}}^{*}-\omega_{d, x^{*} x^{*}}^{*}\right)=0
\end{gathered}
$$

5. Continuity of pressure at water/porous bed interface

$$
P_{2}^{*} K+\rho_{f} g L\left\{\frac{\partial \phi_{1}^{*}}{\partial t^{*}}+\frac{1}{2}\left[2 U^{*} \frac{\partial \phi_{1}^{*}}{\partial x^{*}}+\left(\frac{\partial \phi_{1}^{*}}{\partial x^{*}}\right)^{2}+\left(\frac{\partial \phi_{1}^{*}}{\partial y^{*}}\right)^{2}\right]\right\}=0
$$

6. Far field of porous bed

$$
\lim _{y^{*} \rightarrow-\infty}\left\{\phi_{1}^{*}, \varphi_{d}^{*}, \varphi_{D}^{*}, \omega_{d}^{*}\right\}=0
$$


7. Upstream/downstream boundary

$$
\begin{array}{ll}
\lim _{x^{*} \rightarrow \pm \infty} \frac{\partial}{\partial x^{*}}\left\{\varphi_{d}^{*}, \varphi_{D}^{*}, \omega_{d}^{*}\right\}=0 & \begin{array}{ll}
\frac{\partial \phi_{1}^{*}}{\partial x^{*}} \pm \mathrm{i} k_{0} \phi_{1}^{*}=0, & \text { periodic wave problem } \\
\frac{\partial \phi_{1}^{*}}{\partial x^{*}}=0, & \text { transient wave problem }
\end{array}
\end{array}
$$

In addition, the non-dimensional kinematic boundary condition at the bed surface is

$$
\frac{\partial \gamma^{*}}{\partial t^{*}}=\frac{\partial}{\partial t^{*}}\left[\frac{\partial \varphi_{d}^{*}}{\partial y^{*}}-\frac{\partial \omega_{d}^{*}}{\partial x^{*}}-\frac{\partial \gamma^{*}}{\partial x^{*}}\left(\frac{\partial \varphi_{d}^{*}}{\partial x^{*}}+\frac{\partial \omega_{d}^{*}}{\partial y^{*}}\right)\right]
$$

\section{COMPUTATION AND DISCUSSION}

To estimate the variation of channel bed accurately and effectively, the proposed numerical computation adopts the boundary-fitted co-ordinate system of Thompson et al. [25] to transform the physical domain into the computational domain by substitution of the Dirichlet boundary conditions. Taking the Laplace equations

$$
\begin{aligned}
& \xi_{x x}+\xi_{y y}=0 \\
& \eta_{x x}+\eta_{y y}=0
\end{aligned}
$$

as the grid generator, the orthogonality of grids on the boundaries can be maintained. Besides the orthogonal boundary-fitted co-ordinate transformations, the new equations are expressed in semi-differential form using the finite difference method. Time and space are discretized separately. The time scale is discretized by the first-order backward difference scheme while the space domain is discretized using the central difference method. The computation is divided into channel flow region and porous bed region. These two regions are carried out separately and sequentially, and they are linked by the boundary conditions at the interface. At the moving boundary, the interpolation adopts a cubic spline to keep the shape of porous bed and regenerate the grids to make sure the orthogonality condition is maintained at each time step. The expressions of the independent variables by the finite difference method are written as:

$$
\begin{aligned}
F_{i, j}^{n} & =\frac{5}{2} F_{i+1, j}^{n-1}-\frac{4}{2} F_{i+2, j}^{n-1}+\frac{1}{2} F_{i+3, j}^{n-1} \\
F_{i, j}^{n} & =\frac{5}{2} F_{i-1, j}^{n-1}-\frac{4}{2} F_{i-2, j}^{n-1}+\frac{1}{2} F_{i-3, j}^{n-1}
\end{aligned}
$$

where $F$ is the independent variable for the channel flow or the porous bed, the superscript $n$ refers to the time step and the subscripts $i, j$ are the grid co-ordinates.

In order to verify our formulation, this numerical model without simplification (Equations (37), (39), (41)-(44), along with the boundary conditions equations (47), (49)-(54) and the surface elevation equations (48), (55)) is applied to simulate the problem of a simple periodic wave progression in combination with a constant current. Taking the current 


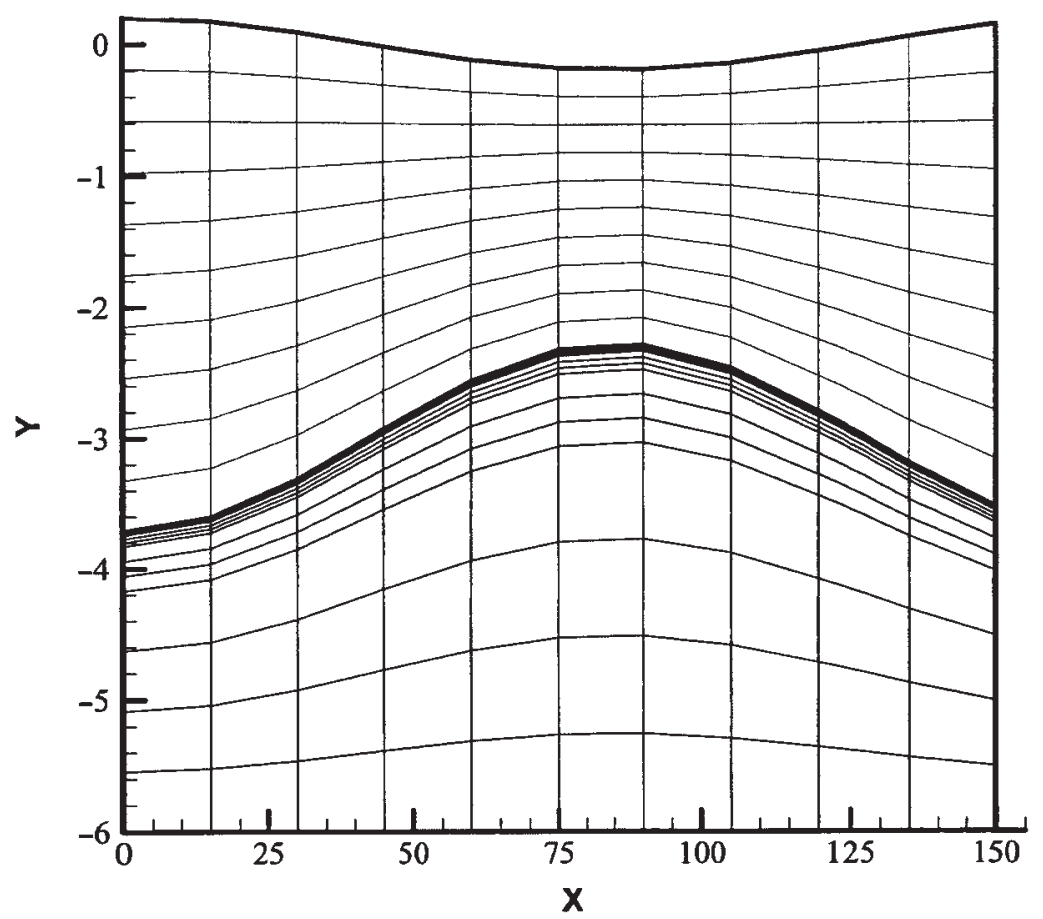

Figure 2. Refined grid near the interface of the computational domain.
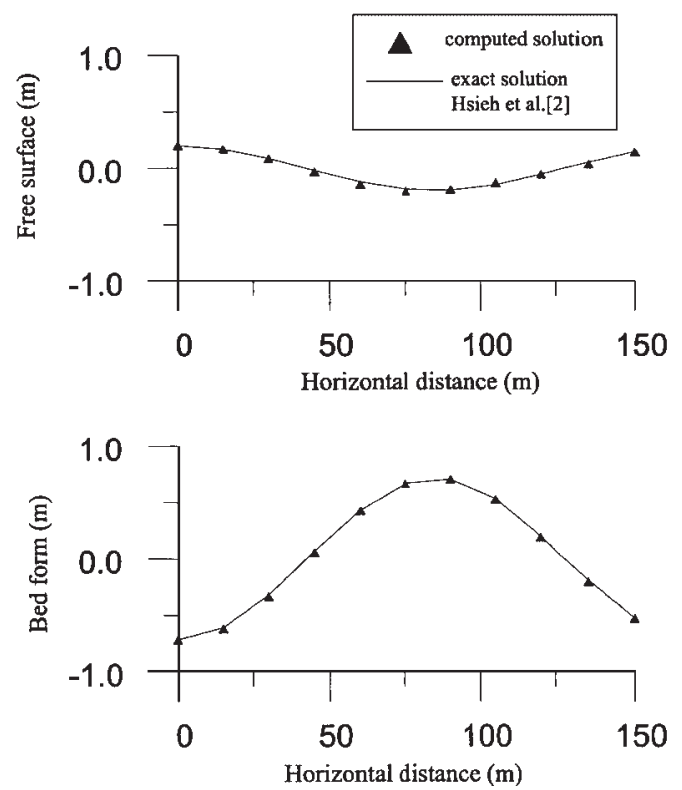

Figure 3. Comparison of computed profiles of free surface $(\zeta)$ and bed form $(\gamma)$ with exact solution. 
$U=0.3 \mathrm{~m} / \mathrm{s}$, water depth $h=3 \mathrm{~m}$, time to warm up $t=60 \mathrm{~s}$, time step $\Delta t=0.1 \mathrm{~s}$ for channel flow, but $0.01 \mathrm{~s}$ for porous bed, and the thickness of channel bed $=3 \mathrm{~m}$, the computed results of free surface and bed form are compared with the analytic solution proposed by Hsieh et al. [20]. Figure 2 shows that the meshes are taken as 10 by 10 grids for region 1, but they are much finer close to the interface with region 2. Figure 3 shows good agreement for the free surface and bed form with the solution of Hsieh et al. [20]. Furthermore, the results for the displacement potentials of both the dilatational and rotational waves at selected sections (indicated by the downward arrows in Figure 4) also agree very well with the Hsieh et al. [20] analytical solutions.
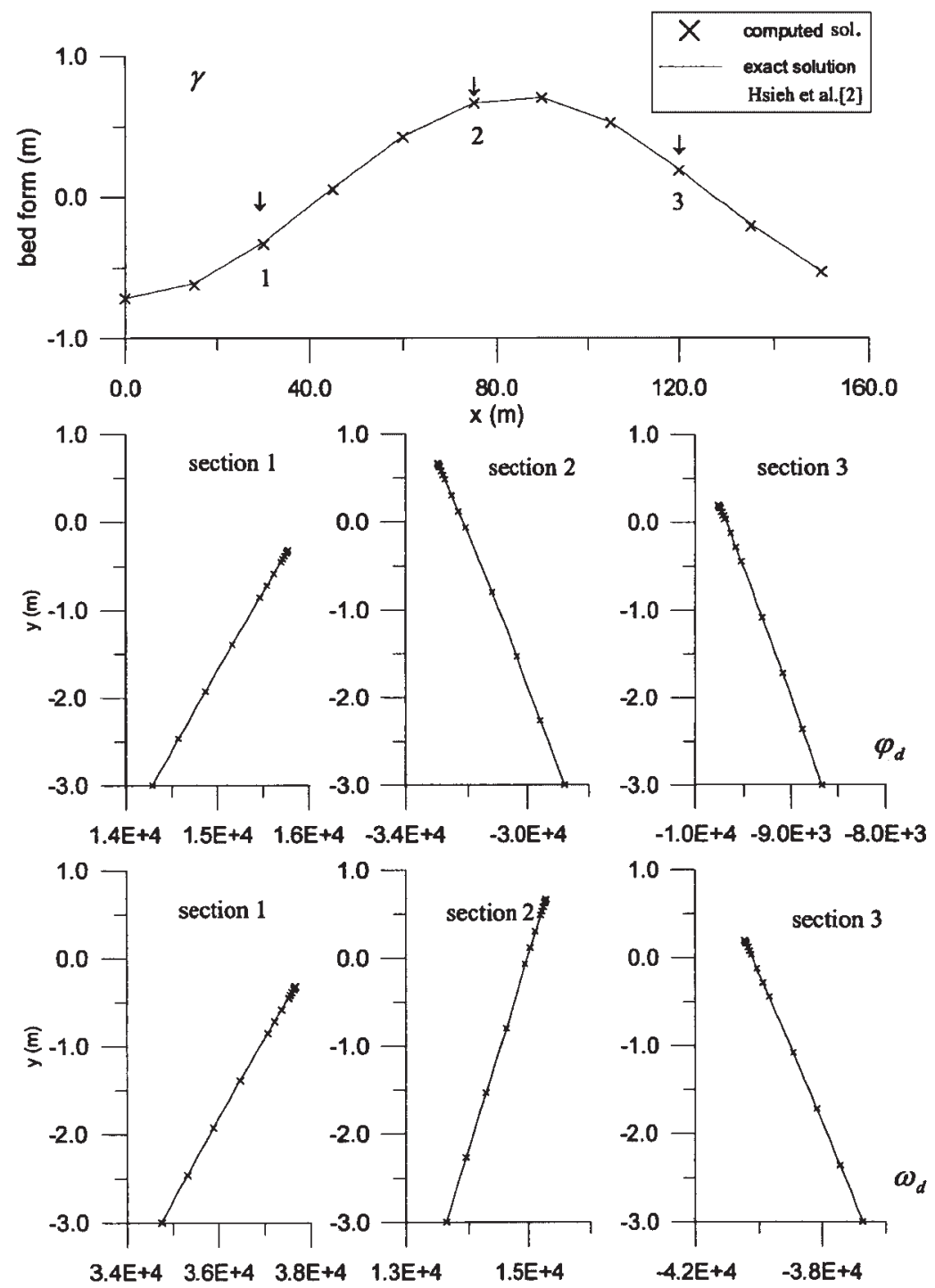

Figure 4. Comparison of computed displacement potentials of dilatational wave $\left(\varphi_{d}\right)$ and rotational wave $\left(\omega_{d}\right)$ with exact solution. 

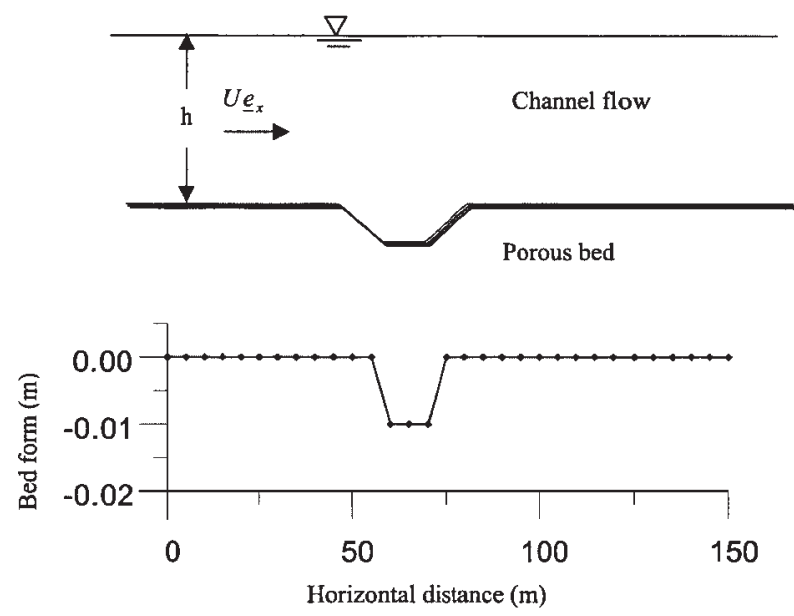

Figure 5. Schematic diagram of a trench at the channel bed.
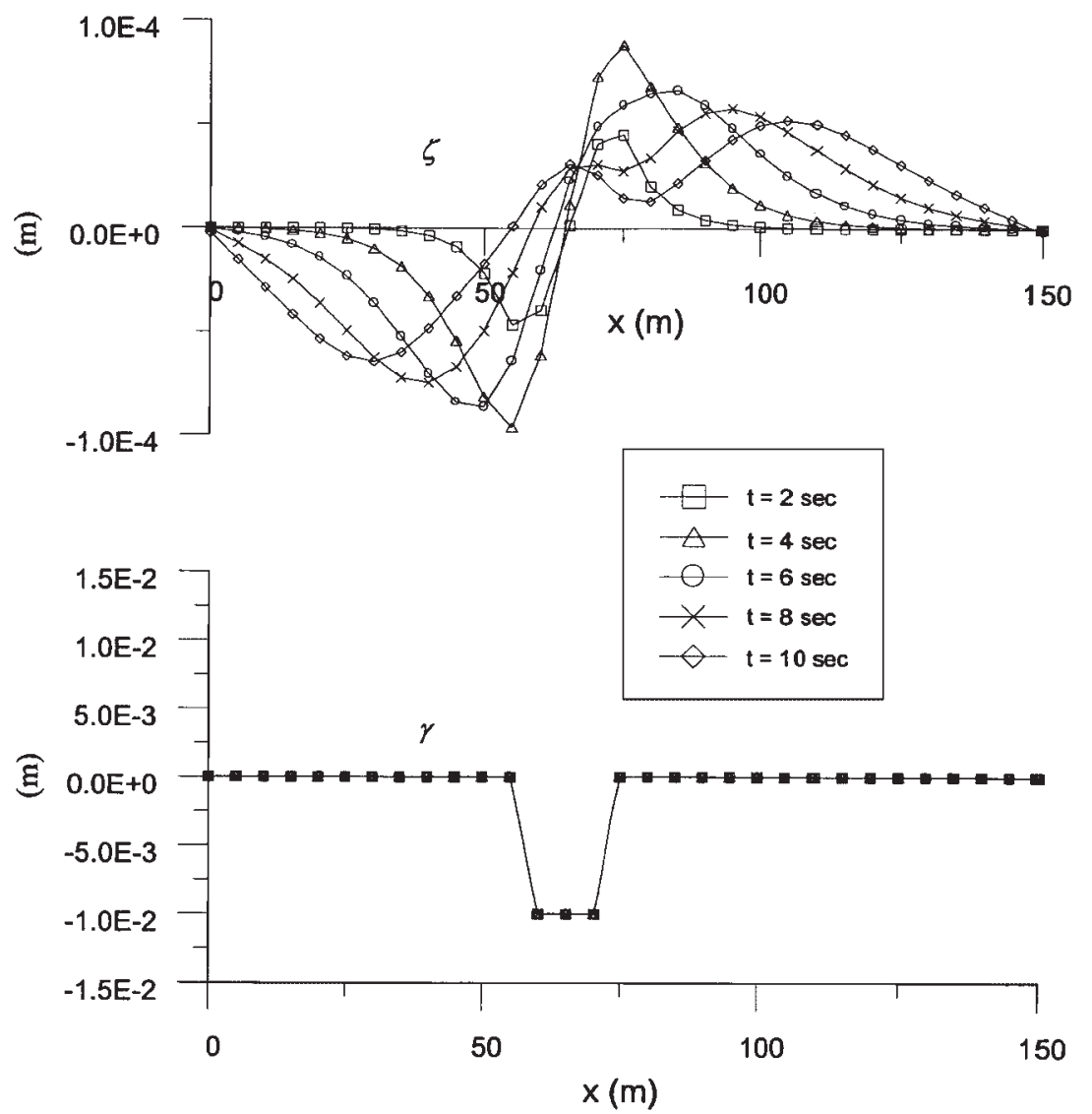

Figure 6. Transient variation of free surface and a trench at bed form, full formulation $(\Delta t=1 \mathrm{~s}, U=0.3 \mathrm{~m} / \mathrm{s})$. 
For the first application, the transient response of a small trench at the channel bed, an important problem of river bed dredging, is simulated. Figure 5 is the schematic diagram of a small trench that is analysed. Figure 6 shows the transient transport of the trench by adopting the original complete formulation (Equations (37), (39), (41), (43), (44) with boundary conditions (47)-(54)). Figure 6 shows that the fluctuation of water surface is very large and continuously increases while the bed form remains unchanged. From the results in Figure 6, we find that although small meshes are used near the interface and the time step is small, the numerical model without simplification is still very unstable and will diverge after a very short period of time, say $10 \mathrm{~s}$. This is because that the dilatational waves for water and solid inside the soft porous bed are almost the same, hence Darcy's terms multiplied by a very large coefficient $b$ (the third terms on the right-hand side of Equations (39) and (41)) are impossible to estimate accurately even after non-dimensionalization. Referring to Hsieh et al. [20], it is noticeable that when the porous bed is soft, the wavelength of the second dilatational wave near the interface is much shorter than that in the homogeneous water, which makes convergence problematic. Thus, the traditional approach of refining meshes near the interface is unsuccessful. However,

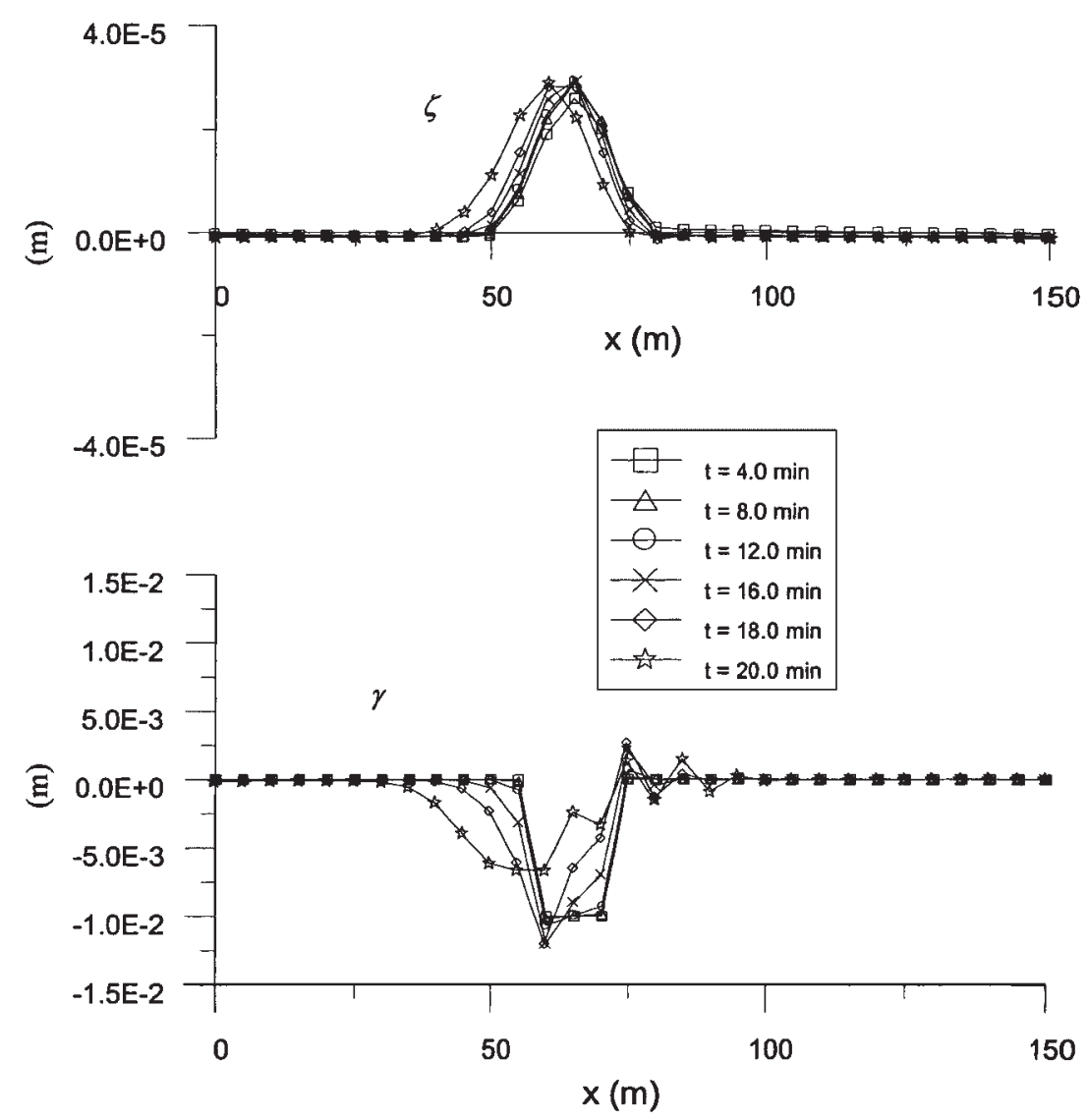

Figure 7. Transient variation of free surface and a trench at bed form, simplified formulation $(\Delta t=60 \mathrm{~s}, U=0.3 \mathrm{~m} / \mathrm{s})$. 


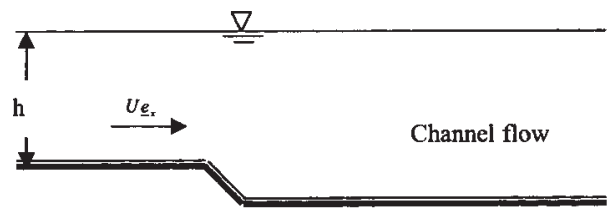

Porous bed

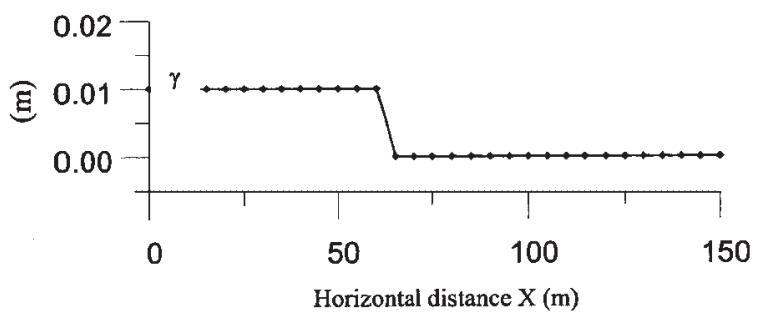

Figure 8. Schematic diagram of channel bed with a downward step.
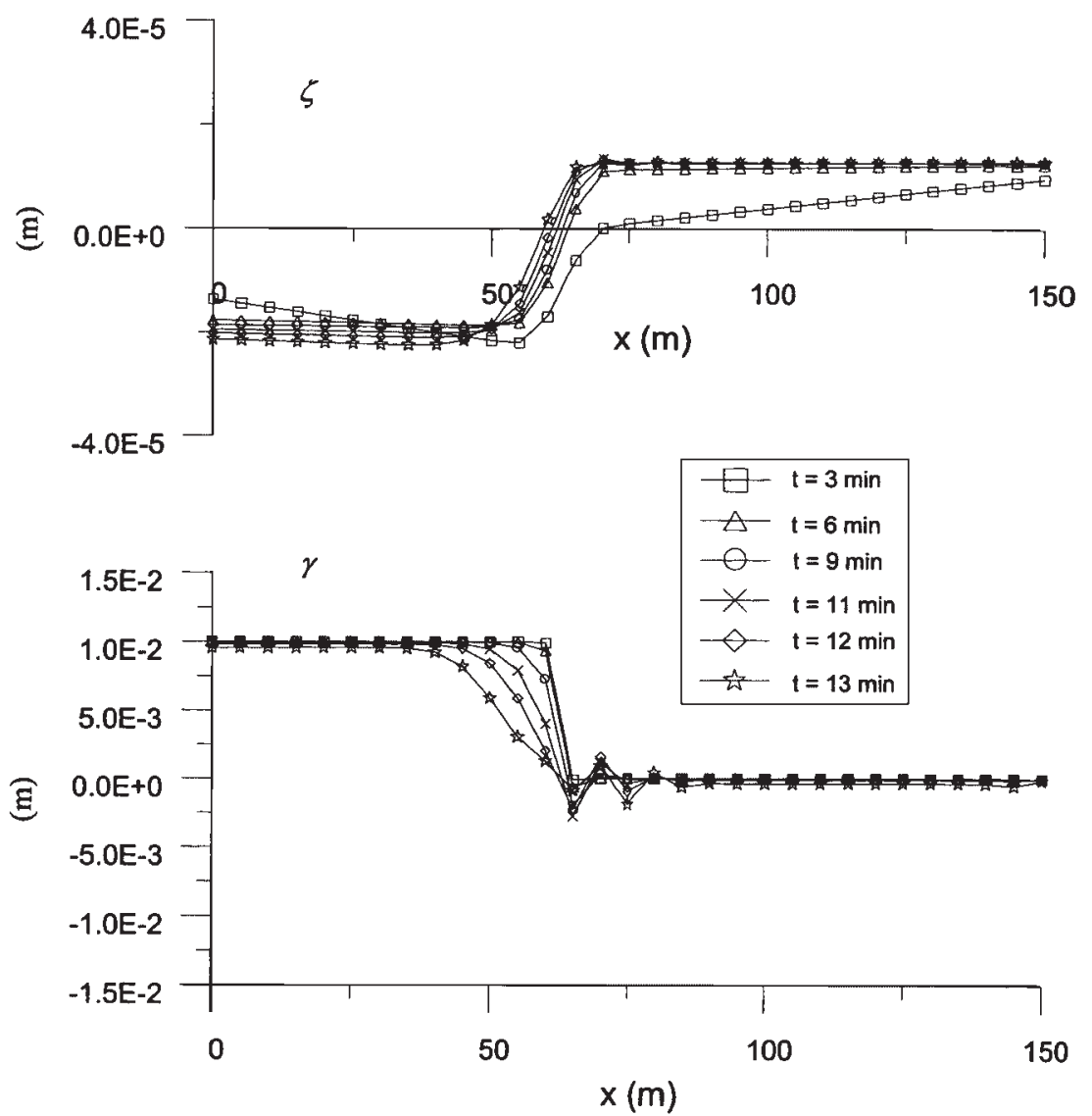

Figure 9. Transient variation of free surface and a downward-step channel bed, simplified formulation $(\Delta t=60 \mathrm{~s}, U=0.3 \mathrm{~m} / \mathrm{s})$. 
the magnitude of the second longitudinal wave is rather small and requires only the so-called boundary layer correction (see Reference [20]). Since Darcy's terms are mainly due to the second longitudinal wave, i.e. the relative velocity of the irrotational part, we find that when the porous material is soft, Darcy's terms are negligible. After neglecting Darcy's terms, Equations (39) and (41) turn out to be Equations (45) and (46). This simplified model is stable, larger time step is allowable, and the meshes near the interface no longer need to be refined.

Indeed, this becomes apparent when the same problem as in Figure 6 is analysed using the simplified procedure. (The finite difference scheme is described in Appendix A.) The results are shown in Figure 7. The time step in Figure 7 is $60 \mathrm{~s}$ instead of $1 \mathrm{~s}$ in Figure 6. After about $12 \mathrm{~min}$, the channel bed begins to deform.

A second application involves the channel bed with a downward step under a uniform flow, as shown in Figure 8. This is an important problem for bridge pier protection applications when river beds are dredged. The transient variations of the free surface and the channel bed are shown in Figure 9, which indicates the deformation of the head-cutting of channel bed together with the upstream going water wave.

\section{CONCLUSION}

The transient response of poroelastic bed form due to stream flow and non-linear water wave are investigated numerically. The theory of potential flow is applied to channel flow while Biot's theory of poroelasticity [21] is adopted to deal with the deformable porous bed. The numerical model without simplification can simulate the periodic wave/current problem with a simple or regular geometry only. But it is easy to diverge within very short time for the transient problem with irregular geometry. The transient problem with irregular geometry always needs small grids near the bed/water interface as well as very small time steps. Even then, convergence is not assured. The present work establishes a simplified numerical model based on the boundary layer concept proposed by Hsieh et al. [20] by neglecting Darcy's terms for a soft poroelastic bed. This makes the study of transient deformations of a channel bed with a trench or a downward step feasible.

\section{ACKNOWLEDGEMENTS}

This study is sponsored by the National Science Council of the Republic of China under grants NSC 86-2621-E002-016 and NSC 88-2611-E-002-028.

\section{APPENDIX A: FINITE DIFFERENCE FORMULATIONS}

Governing equations:

Region 1:

$$
\hat{\alpha}_{1} \phi_{1, \xi \xi}^{*_{n}}+2 \hat{\alpha}_{2} \phi_{1, \xi \eta}^{*_{n}}+\hat{\alpha}_{3} \phi_{1, \eta \eta}^{*_{n}}+\hat{\alpha}_{4} \phi_{1, \xi}^{*_{n}}+\hat{\alpha}_{5} \phi_{1, \eta}^{*_{n}}=0
$$


where

$$
\begin{gathered}
\hat{\alpha}_{1}=\xi_{x^{*}}^{2}+\xi_{y^{*}}^{2} \\
\hat{\alpha}_{2}=\xi_{x^{*}} \eta_{x^{*}}+\xi_{y^{*}} \eta_{y^{*}} \\
\hat{\alpha}_{3}=\eta_{x^{*}}^{2}+\eta_{y^{*}}^{2} \\
\hat{\alpha}_{4}=\xi_{x^{*} x^{*}}+\xi_{y^{*} y^{*}} \\
\hat{\alpha}_{5}=\eta_{x^{*} x^{*}}+\eta_{y^{*} y^{*}}
\end{gathered}
$$

Region 2:

$$
\begin{aligned}
& \frac{2 G+\lambda}{K}\left(\hat{\alpha}_{1} \phi_{d, \xi \xi}^{*_{n}}+2 \hat{\alpha}_{2} \phi_{d, \xi \eta}^{*_{n}}+\hat{\alpha}_{3} \phi_{d, \eta \eta}^{*_{n}}+\hat{\alpha}_{4} \phi_{d, \xi}^{*_{n}}+\hat{\alpha}_{5} \phi_{d, \eta}^{*_{n}}\right)-P_{2}^{*_{n}} \\
& =\Omega_{1} \frac{\phi_{d}^{*_{n}}-2 \phi_{d}^{*{ }_{n-1}}+\phi_{d}^{*{ }_{n-2}}}{\Delta t^{* 2}}+\Omega_{2} \frac{\phi_{D}^{*{ }^{*}}-2 \phi_{D}^{*{ }_{n-1}}+\phi_{D}^{*{ }_{n-2}}}{\Delta t^{* 2}} \\
& -\Omega_{3} P_{2}^{*_{n}}=\Omega_{4} \frac{\phi_{d}^{*_{n}}-2 \phi_{d}^{*{ }_{n-1}}+\phi_{d}^{*_{n-2}}}{\Delta t^{*}{ }_{2}}+\Omega_{5} \frac{\phi_{D}^{*_{n}}-2 \phi_{D}^{*_{n-1}}+\phi_{D}^{*_{n-2}}}{\Delta t^{*_{2}}} \\
& \frac{G}{K}\left(\hat{\alpha}_{1} \omega_{d, \xi \xi}^{*_{n}}+2 \hat{\alpha}_{2} \omega_{d, \xi \eta}^{*_{n}}+\hat{\alpha}_{3} \omega_{d, \eta \eta}^{*_{n}}+\hat{\alpha}_{4} \omega_{d, \xi}^{*_{n}}+\hat{\alpha}_{5} \omega_{d, \eta}^{*_{n}}\right) \\
& =\Omega_{1} \frac{\omega_{d}^{*_{n}}-2 \omega_{d}^{*_{n-1}}+\omega_{d}^{*_{n-2}}}{\Delta t^{*}{ }_{2}}+\Omega_{2} \frac{\omega_{D}^{*_{n}}-2 \omega_{D}^{*_{n-1}}+\omega_{D}^{*_{n-2}}}{\Delta t^{*}{ }_{2}} \\
& 0=\Omega_{4} \frac{\omega_{d}^{* n}-2 \omega_{d}^{* n-1}+\omega_{d}^{*{ }_{n-2}}}{\Delta t^{*}{ }_{2}}+\Omega_{5} \frac{\omega_{D}^{*_{n}}-2 \omega_{D}^{*_{n-1}}+\omega_{D}^{*{ }_{n-2}}}{\Delta t^{*}{ }_{2}} \\
& \frac{P_{2}^{*{ }^{n}}-2 P_{2}^{*{ }^{n-1}}+P_{2}^{n-2}}{\Delta t^{* 2}} \\
& =\frac{1}{n_{0}} \frac{1}{\Delta t^{*}}\left\{( 1 - n _ { 0 } ) \left[\left(\hat{\alpha}_{1} \phi_{d, \xi \xi}^{*{ }^{n} \xi}+2 \hat{\alpha}_{2} \phi_{d, \xi \eta \eta}^{* n}+\hat{\alpha}_{3} \phi_{d, \eta \eta}^{*{ }^{n}}+\hat{\alpha}_{4} \phi_{d, \xi}^{*{ }^{n}}+\hat{\alpha}_{5} \phi_{d, \eta}^{*{ }^{n}}\right)\right.\right. \\
& \left.-\left(\hat{\alpha}_{1} \phi_{d, \xi \xi}^{* n-1}+2 \hat{\alpha}_{2} \phi_{d, \xi \eta}^{* n-1}+\hat{\alpha}_{3} \phi_{d, \eta \eta}^{* n-1}+\hat{\alpha}_{4} \phi_{d, \xi}^{* n-1}+\hat{\alpha}_{5} \phi_{d, \eta}^{* n-1}\right)\right] \\
& +n_{0}\left[\left(\hat{\alpha}_{1} \phi_{D, \xi \xi}^{*_{n}{ }^{n}}+2 \hat{\alpha}_{2} \phi_{D, \xi \eta}^{*_{n} n_{n}}+\hat{\alpha}_{3} \phi_{D, \eta \eta \eta}^{*{ }_{n}}+\hat{\alpha}_{4} \phi_{D, \xi}^{*_{n}}+\hat{\alpha}_{5} \phi_{D, \eta}^{*_{n}}\right)\right. \\
& \left.\left.-\left(\hat{\alpha}_{1} \phi_{D, \xi \xi}^{* n-1}+2 \hat{\alpha}_{2} \phi_{D, \xi \eta}^{* n-1}+\hat{\alpha}_{3} \phi_{D, \eta \eta}^{* n-1}+\hat{\alpha}_{4} \phi_{D, \xi}^{{ }_{n}{ }_{n-1}}+\hat{\alpha}_{5} \phi_{D, \eta}^{* n-1}\right)\right]\right\}
\end{aligned}
$$

where

$$
\begin{gathered}
\Omega_{1}=\frac{\left(\rho_{11}+\rho_{12}\right) g L}{K} \\
\Omega_{2}=\frac{\left(\rho_{12}+\rho_{22}\right) g L}{K} \\
\Omega_{3}=n_{0} \frac{K}{b L^{2}} \sqrt{\frac{L}{g}}
\end{gathered}
$$




$$
\begin{aligned}
& \Omega_{4}=\frac{\rho_{12}}{b} \sqrt{\frac{g}{L}} \\
& \Omega_{5}=\frac{\rho_{22}}{b} \sqrt{\frac{g}{L}}
\end{aligned}
$$

Boundary conditions at the free surface:

(a) Kinematic boundary conditions:

$$
\begin{aligned}
& \left(\phi_{1, \xi^{n}}^{*{ }_{n}} \xi_{y^{*}}+\phi_{1, \eta}^{*{ }_{n}} \eta_{y^{*}}\right) \\
& =\frac{-1}{g}\left\{\frac{\phi_{1}^{*}{ }^{*}-2 \phi_{1}^{*{ }{ }_{n-1}}+\phi_{1}^{*}{ }^{n-2}}{\Delta t^{*}{ }^{2}}+2\left(U^{*}+\phi_{1, \xi}^{{ }^{n}{ }^{n-1}} \xi_{x^{*}}+\phi_{1, \eta}^{{ }^{n-1}} \eta_{x^{*}}\right)\right. \\
& \left(\frac{\phi_{1, \xi}^{*} \xi_{x^{*}}+\phi_{1, \eta}^{*}-\phi_{1, \xi}^{* n-1}-\phi_{1, \eta}^{* n-1} \eta_{x}}{\Delta t^{*}}\right) \\
& +\left(\phi_{1, \xi \xi \xi}^{*} \xi_{x^{*}}^{2}+2 \phi_{1, \xi \eta}^{*}{ }_{n} \xi_{x^{*}} \eta_{x^{*}}+\phi_{1, \eta \eta}^{*}{ }_{n} \eta_{x^{*}}^{2}+\phi_{1, \xi}^{*}{ }_{n}^{n} \xi_{x^{*} x^{*}}+\phi_{1, \eta}^{*}{ }_{n} \eta_{x^{*} x^{*}}\right) \\
& \left(U^{*}+\phi_{1, \eta}^{* n-1} \xi_{x^{*}}+\phi_{1, \eta}^{* n-1} \eta_{x^{*}}\right)^{2}+\left(\phi_{1, \xi}^{* n-1} \xi_{y^{*}} \phi_{1, \eta}^{* n-1} \eta_{y^{*}}\right) \\
& \left(\frac{\phi_{1, \xi}^{*}{ }^{{ }_{n}-1} \xi_{y^{*}}+\phi_{1, \eta}^{*}{ }^{*} \eta_{y^{*}}-\phi_{1, \xi}^{*}{ }^{{ }_{n}-2} \xi_{y^{*}}-\phi_{1, \eta}{ }^{{ }_{n}-2} \eta_{y^{*}}}{\Delta t^{*}}\right) \\
& +\left(\phi_{1, \xi}^{*{ }_{n-1}} \xi_{y^{*}}+\phi_{1, \eta}^{*}{ }_{n-1} \eta_{y^{*}}\right)\left(U^{*}+\phi_{1, \xi}^{*{ }_{n}-1} \xi_{x^{*}}+\phi_{1, \eta}^{*}{ }_{n-1} \eta_{x^{*}}\right) \\
& {\left[\phi_{1, \xi \xi \xi}^{* n-1} \xi_{x^{*}} \xi_{y^{*}}+\phi_{1, \xi \eta}^{* n-1}\left(\xi_{y^{*}} \eta_{x^{*}}+\xi_{x^{*}} \eta_{y^{*}}\right)\right.} \\
& \left.\left.+\phi_{1, \eta \eta}^{* n-1} \eta_{x^{*}} \eta_{y^{*}}+\phi_{1, \xi}^{* n-1} \xi_{x^{*} y^{*}}+\phi_{1, \eta}^{* n-1} \eta_{x^{*} y^{*}}\right]\right\}
\end{aligned}
$$

(b) Dynamic boundary condition:

$$
\begin{aligned}
& \frac{\phi_{1}^{*_{n}}-\phi_{1}^{*{ }_{n-1}}}{\Delta t^{*}}+\frac{1}{2}\left[2 U^{*}\left(\phi_{1, \xi}^{*{ }_{n}} \xi_{x^{*}}+\phi_{1, \eta}^{*} \eta_{x^{*}}\right)+\left(\phi_{1, \xi}^{*{ }_{n}} \xi_{x^{*}}+\phi_{1, \eta}^{*{ }_{n}} \eta_{x^{*}}\right)^{2}\right. \\
& \left.+\left(\phi_{1, \xi}^{*{ }_{n}} \xi_{y^{*}}+\phi_{1, \eta}^{*{ }_{n}} \eta_{y^{*}}\right)^{2}\right]+\zeta^{n}=0
\end{aligned}
$$

Boundary conditions at the porous bed interface:

(a) Continuity of flux:

$$
\begin{aligned}
& \left(\phi_{1, \xi}^{*{ }_{n}} \xi_{y^{*}}+\phi_{1, \eta}^{* n} \eta_{y^{*}}\right)-\frac{y_{\xi}^{*}{ }^{*}{ }^{n-1}}{x_{\xi}^{{ }^{*}{ }^{*}-1}}\left(\phi_{1, \xi}^{*{ }^{*} \xi_{x^{*}}}+\phi_{1, \eta}^{*{ }_{n}} \eta_{x^{*}}\right) \\
& =U^{*} \frac{y_{\xi}^{*}{ }^{*} n-1}{x_{\xi}^{* n-1}}+\frac{1}{\Delta t^{*}}\left\{\left[\left(1-n_{0}\right)\left(1-\frac{y_{\xi}^{*{ }^{n}-1}}{x_{\xi}^{*}{ }^{*}{ }^{*}}\right)\left(\varphi_{d, \xi}^{*_{n}} \xi_{y^{*}}+\varphi_{d, \eta}^{*_{n}} \eta_{y^{*}}-\omega_{d, \xi}^{*_{n}} \xi_{x^{*}}-\omega_{d, \eta}^{*_{n}} \eta_{x^{*}}\right)\right.\right.
\end{aligned}
$$




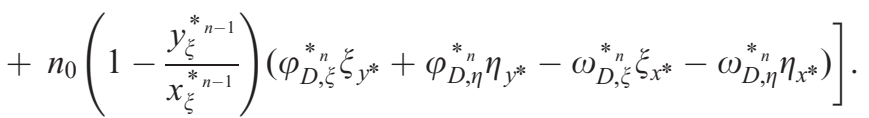

$$
\begin{aligned}
& -\left[\left(1-n_{0}\right)\left(1-\frac{y_{\xi}^{*}{ }^{*}-2}{x_{\xi}^{*}{ }^{n-2}}\right)\left(\varphi_{d, \xi}^{*_{n-1}} \xi_{y^{*}}+\varphi_{d, \eta}^{* n-1} \eta_{y^{*}}-\omega_{d, \xi}^{{ }^{n-1}} \xi_{x^{*}}-\omega_{d, \eta}^{* n-1} \eta_{x^{*}}\right)\right. \\
& \left.\left.+n_{0}\left(1-\frac{y_{\xi}^{*}{ }^{n-2}}{x_{\xi}^{* n-2}}\right)\left(\varphi_{D, \xi}^{* n-1} \xi_{y^{*}}+\varphi_{D, \eta}^{* n-1} \eta_{y^{*}}-\omega_{D, \xi}^{* n-1} \xi_{x^{*}}-\omega_{D, \eta}^{* n-1} \eta_{x^{*}}\right)\right] \cdot\right\}
\end{aligned}
$$

(b) Continuity of effective stress:

$$
\begin{aligned}
& \frac{G}{K}\left\{2 \left[\varphi_{d, \xi \xi \xi}^{*_{n}} \xi_{x^{*}} \xi_{y^{*}}+\varphi_{d, \xi \eta}^{*_{n}}\left(\xi_{x^{*}} \eta_{y^{*}}+\xi_{y^{*}} \eta_{x^{*}}\right)\right.\right. \\
& \left.+\varphi_{d, \eta \eta}^{*}{ }_{n}^{*} \eta_{x^{*}} \eta_{y^{*}}+\varphi_{d, \xi}^{*{ }_{n}} \xi_{x^{*} y^{*}}+\varphi_{d, \eta}^{*}{ }_{n}^{n} \eta_{x^{*} y^{*}}\right] \\
& +\left[\omega_{d, \xi \xi}^{{ }^{*}{ }^{n} \xi^{*}} \xi_{y^{*}}^{2}+2 \omega_{d, \xi \eta}^{* n} \xi_{y^{*}} \eta_{y^{*}}+\omega_{d, \eta \eta}^{*} \eta_{y^{*}}^{2}+\omega_{d, \xi}^{{ }^{*}{ }^{n}} \xi_{y^{*} y^{*}}+\omega_{d, \eta}^{* n} \eta_{y^{*} y^{*}}\right] \\
& \left.+\left[\omega_{d, \xi^{n} \xi}^{*_{n}} \xi_{x^{*}}^{2}+2 \omega_{d, \xi \eta}^{*_{n}} \xi_{x^{*}} \eta_{x^{*}}+\omega_{d, \eta \eta}^{*} \eta_{x^{*}}^{2}+\omega_{d, \xi}^{*_{n}} \xi_{x^{*} x^{*}}+\omega_{d, \eta}^{*} \eta_{x^{*} x^{*}}\right]\right\}
\end{aligned}
$$

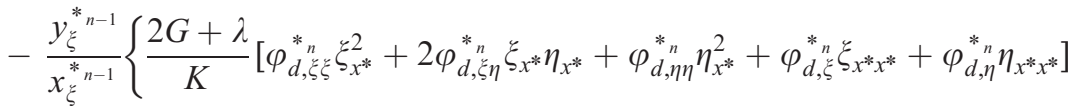

$$
\begin{aligned}
& +2 \frac{G}{K}\left[\omega_{d, \xi \xi \xi}^{*_{n}^{n}} \xi_{x^{*}} \xi_{x_{y^{*}}}+\omega_{d, \xi \eta}^{*_{n}}\left(\xi_{x^{*}} \eta_{y^{*}}+\xi_{y^{*}} \eta_{x^{*}}\right)\right. \\
& \left.+\omega_{d, \eta \eta}^{*} \eta_{x^{*}} \eta_{y^{*}}+\omega_{d, \xi^{n}}^{{ }^{n} \xi_{x^{*} y^{*}}}+\omega_{d, \eta}^{*{ }_{n}} \eta_{x^{*} y^{*}}\right]
\end{aligned}
$$

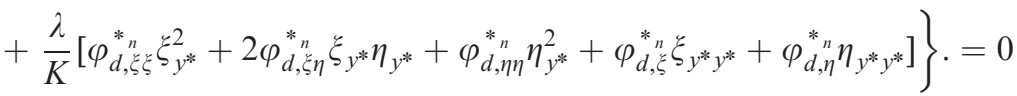

$$
\begin{aligned}
& \left\{\frac{2 G+\lambda}{K}\left[\varphi_{d, \xi^{n} \xi}^{{ }_{n}} \xi_{y^{*}}^{2}+2 \varphi_{d, \xi \eta}^{*_{n}} \xi_{y^{*}} \eta_{y^{*}}+\varphi_{d, \eta \eta}^{{ }_{n}} \eta_{y^{*}}^{2}+\varphi_{d, \xi}^{{ }_{n}{ }_{n} \xi_{y^{*} y^{*}}}+\varphi_{d, \eta}^{*} \eta_{y^{*} y^{*}}\right]\right. \\
& -2 \frac{G}{K}\left[\omega_{d, \xi \xi \xi}^{* n} \xi_{x^{*}} \xi_{y^{*}}+\omega_{d, \xi \eta}^{*}\left(\xi_{x^{*}} \eta_{y^{*}}+\xi_{y^{*}} \eta_{x^{*}}\right)\right. \\
& \left.+\omega_{d, \eta \eta \eta}^{* n} \eta_{x^{*}} \eta_{y^{*}}+\omega_{d, \xi}^{* n} \xi_{x^{*} y^{*}}+\omega_{d, \eta}^{*}{ }_{n}^{n} \eta_{x^{*} y^{*}}\right] \\
& \left.+\frac{\lambda}{K}\left[\varphi_{d, \xi \xi}^{*_{n} \xi_{x^{*}}}+2 \varphi_{d, \xi \eta}^{*_{n}} \xi_{x^{*}} \eta_{x^{*}}+\varphi_{d, \eta \eta}^{*_{n}} \eta_{x^{*}}^{2}+\varphi_{d, \xi}^{*_{n} \xi_{x^{*} x^{*}}}+\varphi_{d, \eta}^{*_{n}} \eta_{x^{*} x^{*}}\right]\right\} . \\
& -\frac{y_{\xi}^{*}{ }^{n-1}}{x_{\xi}^{* n-1}} \frac{G}{K}\left\{2 \left[\varphi_{d, \xi^{*} \xi^{*}}^{*} \xi_{x^{*}} \xi_{y^{*}}+\varphi_{d, \xi \eta}^{* n}\left(\xi_{x^{*}} \eta_{y^{*}}+\xi_{y^{*}} \eta_{x^{*}}\right)\right.\right. \\
& \left.+\varphi_{d, \eta \eta \eta}^{*} \eta_{x^{*}} \eta_{y^{*}}+\varphi_{d, \xi^{n}}^{{ }_{n}{ }^{n}} \xi_{x^{*} y^{*}}+\varphi_{d, \eta}^{*}{ }_{n} \eta_{x^{*} y^{*}}\right]
\end{aligned}
$$

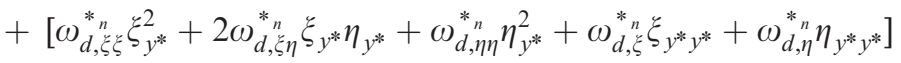

$$
\begin{aligned}
& \left.+\left[\omega_{d, \xi \xi}^{* n} \xi_{x^{*}}^{2}+2 \omega_{d, \xi \eta}^{* n} \xi_{x^{*}} \eta_{x^{*}}+\omega_{d, \eta \eta}^{*}{ }_{n}^{n} \eta_{x^{*}}^{2}+\omega_{d, \xi}^{*_{n} \xi_{x^{*} x^{*}}}+\omega_{d, \eta}^{*} \eta_{x^{*} x^{*}}\right]\right\}=0
\end{aligned}
$$


(c) Continuity of pressure:

$$
\begin{aligned}
& P_{2}^{{ }^{*} n} K+\rho_{f} g L\left\{\frac{\phi_{1}^{*}{ }^{*}-\phi_{1}^{* n-1}}{\Delta t^{*}}+\frac{1}{2}\left[2 U^{*}\left(\phi_{1, \xi}^{*{ }_{n}} \xi_{x^{*}}+\phi_{1, \eta}^{*{ }_{n}} \eta_{x^{*}}\right)\right.\right. \\
& \left.\left.+\left(\phi_{1, \xi}^{*_{n}} \xi_{x^{*}}+\phi_{1, \eta}^{*} \eta_{x^{*}}\right)^{2}+\left(\phi_{1, \xi}^{*_{n}} \xi_{y^{*}}+\phi_{1, \eta}^{*} \eta_{y^{*}}\right)^{2}\right]\right\} \cdot=0
\end{aligned}
$$

(d) Far field of porous bed:

$$
\phi_{1}^{*}=\varphi_{d}^{*}=\varphi_{D}^{*}=\omega_{d}^{*}=\omega_{D}^{*}=0
$$

(e) Upstream boundary:

$$
\begin{gathered}
\phi_{1 i, j}^{*_{n}}=\frac{5}{2} \phi_{1 i+1, j}^{*_{n-1}}-\frac{4}{2} \phi_{1 i+2, j}^{*_{n-1}}+\frac{1}{2} \phi_{1 i+3, j}^{*_{n-1}} \\
5 \varphi_{d i+1, j}^{*_{n}}-4 \varphi_{d i+2, j}^{*_{n}}+\varphi_{d i+3, j}^{*_{n}}=0 \\
5 \varphi_{D i+1, j}^{*_{n}}-4 \varphi_{D i+2, j}^{*_{n}}+\varphi_{D i+3, j}^{*_{n}}=0 \\
5 \omega_{d i+1, j}^{*_{n}}-4 \omega_{d i+2, j}^{*_{n}}+\omega_{d i+3, j}^{*_{n}}=0
\end{gathered}
$$

(f) Downstream boundary:

$$
\begin{gathered}
\phi_{1 i, j}^{*_{n}}=\frac{5}{2} \phi_{1 i-1, j}^{*_{n-1}}-\frac{4}{2} \phi_{1 i-2, j}^{*_{n-1}}+\frac{1}{2} \phi_{1 i-3, j}^{*_{n-1}} \\
5 \varphi_{d i-1, j}^{*_{n}}-4 \varphi_{d i-2, j}^{*_{n}}+\varphi_{d i-3, j}^{*_{n}}=0 \\
5 \varphi_{D i-1, j}^{*_{n}}-4 \varphi_{D i-2, j}^{*_{n}}+\varphi_{D i-3, j}^{*_{n}}=0 \\
5 \omega_{d i-1, j}^{*_{n}}-4 \omega_{d i-2, j}^{*_{n}}+\omega_{d i-3, j}^{*_{n}}=0
\end{gathered}
$$

After the solutions are obtained, the variation of bed form can be found as

$$
\begin{aligned}
& \gamma^{*}{ }_{n}=\gamma^{*}{ }_{n-1}+\left[\left(\varphi_{d, \xi}^{*}{ }_{n} \xi_{y^{*}}+\varphi_{d, \eta}^{*} \eta_{y^{*}}-\omega_{d, \xi}^{*{ }_{n} \xi_{x^{*}}}-\omega_{d, \eta}^{*{ }_{n}} \eta_{x^{*}}\right)\right. \\
& \left.-\frac{y_{\xi}^{*}{ }^{*}{ }^{n-1}}{x_{\xi}^{* n-1}}\left(\varphi_{d, \xi^{n}}^{*{ }^{n}} \xi_{x^{*}}+\varphi_{d, \eta}^{* n} \eta_{x^{*}}+\omega_{d, \xi}^{*_{n}^{n}} \xi_{y^{*}}+\omega_{d, \eta}^{* n} \eta_{y^{*}}\right)\right] \\
& -\left[\left(\varphi_{d, \xi}^{* n-1} \xi_{y^{*}}+\varphi_{d, \eta}^{* n-1} \eta_{y^{*}}-\omega_{d, \xi}^{*{ }^{n-1}} \xi_{x^{*}}-\omega_{d, \eta}^{* n-1} \eta_{x^{*}}\right)\right. \\
& \left.-\frac{y_{\xi}^{* n-2}}{x_{\xi}^{* n-2}}\left(\varphi_{d, \xi}^{* n-1} \xi_{x^{*}}+\varphi_{d, \eta}^{* n-1} \eta_{x^{*}}+\omega_{d, \xi}^{* n-1} \xi_{y^{*}}+\omega_{d, \eta}^{* n-1} \eta_{y^{*}}\right)\right] \text {. }
\end{aligned}
$$

\section{REFERENCES}

1. Graf WH. Hydraulics of Sediment Transport. Table 11.1 and Figure 11.2. McGraw-Hill: New York, 1971; 277.

2. Shibayama T. Sediment transport mechanism and two-dimensional beach transformation due to waves. $D$. Eng. Dissertation, University of Tokyo, Tokyo, 1984.

3. Horikawa K (ed.). Nearshore Dynamics and Coastal Processes. Figure 3.2. University of Tokyo Press: Tokyo, 1988; 172.

4. Lyne WH. Unsteady viscous flow over a wavy wall. Journal of Fluid Mechanics 1971; 50:33-48. 
5. Sleath JFA. A numerical study of the influence of bottom roughness on mass transport by water waves. Proceedings of the International Conference on Numerical Methods Fluid Dynamics, Southampton, 1973.

6. Longuet-Higgins MS. Oscillating flow over steep sand ripples. Journal of Fluid Mechanics 1981; 107:1-35.

7. Vittori G, Blondeaux P. Sand ripples under sea waves-Part 3. Brick-pattern ripple formation. Journal of Fluid Mechanics 1992; 239:23-45.

8. Darwin GH. On the formation of ripple-marks. Proceedings of the Royal Society, London, 1884; 36:18-43.

9. Bagnold RA. The movement of the desert sand. Proceedings of the Royal Society, London Series A 1936; 892(157):594-620.

10. Exner FM. Über die wechselwirkung zwischen wasser und geschiebe in flussen. Sitzungsberichte der Akademie der Wissenschaften. Wein, Heft 3-4, 1925.

11. Anderson AG. The characteristics of sediment waves formed by flow in open channels. Proceedings of the Third Midwestern Conference in Fluid Mechanics, Minneapolis, March 1953; 379-395.

12. Kennedy JF. The mechanics of dunes and antidunes in erodible-bed channels. Journal of Fluid Mechanics 1963; 16:521-544.

13. Biot MA. Theory of propagation elastic waves in a fluid saturated porous solid. I. Low-frequency range. Journal of American Statistical Association 1956; 28:168-178.

14. Mei CC, Foda MA. Wave-induced responses in a fluid filled poroelastic solid with a free surface-a boundary layer theory. Geophysical Journal of the Royal Astronomical Society 1981; 66:597-631.

15. Huang LH, Song CH. Dynamic response of poroelastic bed to water waves. Journal of Hydraulic Engineering, ASCE 1993; 119:1003-1020.

16. Huang LH, Chwang AT. Trapping and absorption of sound waves. II: a sphere covered with a porous layer. Wave Motion 1990; 12:401-414.

17. Chen TW, Huang LH, Song CH. Dynamic response of poroelastic bed to nonlinear water waves. Journal of Engineering Mechanics, ASCE 1997; 123(10):1041-1049.

18. Huang LH, Chiang YL. Bed form formation in alluvial channels. Proceedings of the Seventh International Symposium of River Sedimentation, Hong Kong, 1998.

19. Huang LH, Hsieh PC, Wang TW. Linear oscillatory water wave propagating over soft poroelastic bed. Biot Conference on Poromechanics, Louvain-la-Neuve, Belgium, 1998.

20. Hsieh PC, Huang LH, Wang TW. Dynamic response of soft poroelastic bed to nonlinear waver wave-boundary layer correction approach. Journal of Engineering Mechanics, ASCE 2000; 126(10):1064-1073.

21. Biot MA. Mechanics of deformation and acoustic propagation in porous media. Journal of Applied Physics 1962; 33(4): 1482-1498

22. Verruijt A. In Elastic Storage of Aquifers-Flow through Porous Media, De DeWiest RJM (ed.). Academic Press: New York, 1969.

23. Morse PM, Feshbach H. Method of Theoretical Physics. McGraw-Hill: New York, 1978.

24. Madson OS. Wave-induced pore pressure and effective stress in a porous bed. Geotechnology 1978; 28:377-393.

25. Thompson JF, Thames FC, Mastin CW. Automatical numerical generation of body-fitted curvilinear coordinate systems for fields containing any number of arbitrary two-dimensional bodies. Journal of Computational Physics $1974 ; 15: 299-317$. 